\title{
THE CONJUGATE FOURIER-STIELTJES INTEGRAL IN THE PLANE ${ }^{1}$
}

BY VICTOR L. SHAPIRO

Communicated by S. Bochner, September 27, 1958

Let $K(x)$ with $x=\left(x_{1}, x_{2}\right)$ be a Lip $(\alpha, 2)$ conjugate CalderonZygmund kernel with $1 / 2<\alpha<1$, i.e. $K(x)=\Omega(\theta) r^{-2}$ where $(r, \theta)$ are the usual polar coordinates of $x$ with $\Omega(\theta)$ a continuous periodic function of period $2 \pi$ with vanishing integral over the interval $[0,2 \pi]$ satisfying the condition $\int_{0}^{2 \pi}[\Omega(\theta+h)-\Omega(\theta)]^{2} d \theta=0\left(h^{2 \alpha}\right)$ as $h \rightarrow 0$ (See [2] and [7, p. 106].) Let $F$ be a countably additive set function defined on the Borel sets of the plane having finite total variation. Furthermore let $f(y)=(2 \pi)^{-2} \int_{E_{2}} e^{-i(y, x)} d F(x)$ be the Fourier-Stieltjes transform of $F$ with $E_{2}$ the plane and $(y, x)$ the usual scalar product. Also let $k(y)$ be the principal-valued Fourier transform of $K$, i.e. $k(y)=(2 \pi)^{-2} \lim _{t \rightarrow 0 ; \lambda \rightarrow \infty} \int_{D(0, \lambda)-D(0, t)} e^{-i(y, x)} K(x) d x$ where $D(x, t)$ represents the open disc with center $x$ and radius $t$. (It follows from the above assumptions that $k(y)$ exists for every $y$.) Then formally the conjugate Fourier-Stieltjes integral of $F$ is given by $4 \pi^{2} \int_{E_{2}} e^{i(y, x)} f(y) k(y) d y$. In [2, p. 118], it is shown that $\lim _{t \rightarrow 0} \int_{E_{2}-D_{2}(x, t)} K(x-y) d F(y)$ exists and is finite almost everywhere. We call this limit the conjugate of $F$ with respect to $K$ and designate it by $\widetilde{F}(x)$. With $|y|=\left(y_{1}^{2}+y_{2}^{2}\right)^{1 / 2}$ and $I_{R}(x)=4 \pi^{2} \int_{E_{2}} e^{-|y| / R} e^{i(y, x)} f(y) k(y) d y$, we propose to prove in this note the following theorem:

THEOREM 1. $\lim _{R \rightarrow \infty} I_{R}(x)=\widetilde{F}(x)$ almost everywhere.

In a certain sense this result is the planar analogue of $[7$, p. 54]. In a forthcoming paper we shall extend this result to $n$-dimensional Euclidean space and the $n$-dimensional torus. We shall also study those kernels which are Bochner-Riesz summable almost every where. In particular we shall show that if $K(x)$ is in $C^{\infty}$ then the conjugate Fourier-Stieltjes integral of $F$ is summable $(R, \alpha)$ for $\alpha>1 / 2$ to $\widetilde{F}(x)$ almost everywhere, thus paralleling Bochner's result [1] for the Fourier-Stieltjes integral of $F$.

Letting $D_{\text {sym }} F$ designate the symmetric derivative of $F[5, \mathrm{p} .149]$ and $\int_{B}|d F|$ the total variation of $F$ over $B$, we observe from [5, p. 119 and p. 152] and the standard argument of Lebesgue that

1 This research was supported by the United States Air Force through the Air Force Office of Scientific Research of the Air Research and Development Command under contract No. AF 18(600)-1595. 


$$
\lim _{t \rightarrow 0}\left(\pi t^{2}\right)^{-1} \int_{D(x, t)}\left|d F(y)-D_{s y m} F(x) d y\right|=0 \text { a.e. }
$$

So to prove the above theorem it is sufficient to prove the following theorem:

TheOREM 2. At every point $x$ for which (1) holds

$$
\lim _{R \rightarrow \infty}\left[I_{R}(x)-\int_{E_{2}-D\left(x, R^{-1}\right)} K(x-y) d F(y)\right]=0 .
$$

To prove Theorem 2 , we set $H_{n}(R)=n^{-1} \int_{0}^{\infty} e^{-t / R} J_{n}(t) t d t$ where $J_{n}(t)$ is a Bessel function of the first kind of order $n$ and establish the following lemmas:

Lemma 1. For $n=1,2, \cdots$, and all $R>0$,

(i) $\left|H_{n}(R)\right| \leqq R^{2}$,

(ii) $0<H_{n}(R) \leqq 1$,

(iii) there is a constant $A$ independent of $n$ and $R$ such that

$$
\left|H_{n}(R)-1\right| \leqq A\left[\left(n R^{-1}\right)^{3 / 2}+\left(n R^{-1}\right)^{1 / 2}\right] .
$$

(i) follows immediately from the fact that $\left|J_{n}(t)\right| \leqq 1$. For $n \geqq 2$, (ii) follows on using Euler's integral representation for hypergeometric functions $[6$, p. 384] and $[4$, p. 59], for then

$$
\begin{aligned}
H_{n}(R)= & \Gamma\left(\frac{n}{2}\right) \pi^{-1 / 2}\left[\Gamma\left(\frac{n}{2}-\frac{1}{2}\right)\right]^{-1} \\
& \cdot \int_{0}^{1} t^{n / 2+1 / 2}(1-t)^{n / 2-3 / 2}\left[t+R^{-2}\right]^{-(n / 2+1)} d t .
\end{aligned}
$$

(iii) follows from the fact that for $n \geqq 2$ and $R>2$, there is a constant $A_{1}$ independent of $n$ and $R$ such that

$$
\left|H_{n}(R)-1\right| \leqq A_{1} n^{1 / 2}\left[\int_{0}^{R^{-1}} t^{-1 / 2} d t+n R^{-2} \int_{R^{-1}}^{1 / 2} t^{-3 / 2} d t+n R^{-2}\right] .
$$

For $n=1$, (ii) and (iii) follow from the fact that $H_{1}(R)=\left(1+R^{-2}\right)^{-3 / 2}$.

Lemma 2. Let $\Omega(\theta)=\sum_{n=1}^{\infty} a_{n} e^{i n \theta}+\tilde{a}_{n} e^{-i n \theta}$ and $\alpha>\beta>1 / 2$. Then there is a constant $A$ independent of $R$ and such that for $R \geqq 1$,

$$
\sum_{n=1}^{\infty}\left|a_{n} e^{i n \theta}+\bar{a}_{n} e^{-i n \theta}\right|\left|H_{n}(R)-1\right|<A R^{1 / 2-\beta} .
$$

To prove the lemma, we observe that by $\left[7\right.$, p. 143], $\sum_{n=1}^{\infty} n^{\beta-1 / 2}$ $\cdot\left|a_{n}\right|<A_{1}<\infty$ (consequently $\Omega(\theta)$ is in $\operatorname{Lip} \beta-1 / 2$ ), and therefore 
by Lemma 1 that the sum in the left part of (3) is majorized by a constant multiple of

$$
R^{1 / 2-\beta}\left(\sum_{n=1}^{[R]} 2\left|a_{n}\right| n^{\beta-1 / 2}+\sum_{n=[R]+1}^{\infty} 4\left|a_{n}\right| n^{\beta-1 / 2}\right) \leqq 4 A_{1} R^{1 / 2-\beta}
$$

To prove Theorem 2, we can assume with no loss of generality that $x$ is the origin. Next we see [3, Lemma 2] that for $y \neq 0, k(y)$ $=\sum_{n=1}^{\infty}(2 \pi)^{-1}\left(a_{n} e^{i n \theta}+\bar{a}_{n} e^{-i n \theta}\right)(-i)^{n} n^{-1}$ and consequently that

$$
I_{R}(0)=\int_{E_{2}}\left[\sum_{n=1}^{\infty}\left(a_{n} e^{i n \theta}+\bar{a}_{n} e^{-i n \theta}\right)(-1)^{n} H_{n}(R|u|)\right]|u|^{-2} d F(u) .
$$

Therefore using (1), (i) of Lemma 1, and the absolute convergence of the Fourier series of $\Omega$, we obtain that

$$
\begin{aligned}
\int_{D\left(0, R^{-1}\right)}\left[\sum_{n=1}^{\infty}\left(a_{n} e^{i n \theta}+\tilde{a}_{n} e^{-i n \theta}\right)(-1)^{n} H_{n}(R|u|)\right]|u|^{-2} d F(u) & \\
& =o(1) \text { as } R \rightarrow \infty .
\end{aligned}
$$

Using (ii) of Lemma 1, the absolute convergence of the Fourier series of $\Omega$, and the fact that $F$ is of finite total variation on the plane, we conclude from (4) that to prove the theorem it is sufficient to show that for fixed $\lambda>0$,

(5) $\int_{D(0, \lambda)-D\left(0, R^{-1}\right)}\left[\sum_{n=1}^{\infty}(-1)^{n}\left(a_{n} e^{i n \theta}+\bar{a}_{n} e^{-i n \theta}\right)\left(H_{n}(R|u|)-1\right)\right]$

$$
\cdot|u|^{-2}\left[d F(u)-D_{s y m} F(0) d u\right]=o(1) \text { as } R \rightarrow \infty .
$$

Letting $G(t)=\int_{D(0, t)}\left|d F(u)-D_{\text {sym }} F(0) d u\right|$, we see from Lemma 2 that the left side of (5) is majorized by a constant multiple of

$$
R^{1 / 2-\beta} \int_{R^{-1}}^{\lambda} t^{1 / 2-(2+\beta)} d G(t)
$$

Since by assumption $G(t)=o\left(t^{2}\right)$ as $t \rightarrow 0$, we obtain that (6) is $O\left(R^{1 / 2-\beta}\right)+o(1)+R^{1 / 2-\beta} \int_{R^{-1}}^{\lambda} o\left(t^{2}\right) t^{1 / 2-(3+\beta)} d t$. Consequently (5) is established and the proof of the theorem is complete.

\section{REFERENCES}

1. S. Bochner, Summation of multiple Fourier series by spherical means, Trans. Amer. Math. Soc. vol. 40 (1936) pp. 175-207. 
2. A. P. Calderon and A. Zygmund, On the existence of certain singular integrals, Acta. Math. vol. 88 (1952) pp. 85-139.

3. - On a problem of Mihlin, Trans. Amer. Math. Soc. vol. 78 (1955) pp. 209-224.

4. A. Erdelyi, W. Magnus, F. Oberhettinger, F. G. Tricomi, Higher transcendental functions, vol. 1, New York, 1953.

5. S. Saks, Theory of the integral, Warsaw, 1937.

6. G. N. Watson, A treatise on the theory of Bessel functions, Cambridge, 1952.

7. A. Zygmund, Trigonometrical series, Warsaw, 1935.

\section{RUTGERS UNIVERSITY AND}

The Institute for Advanced Study 